$\sqrt{2}$

CHOICE

\title{
Twenty years experience of selective secondary ultrasound screening for congenital dislocation of the hip
}

\author{
Nicholas M P Clarke, ${ }^{1}$ Isabel C Reading, ${ }^{2}$ Charles Corbin, ${ }^{3}$ Colm C Taylor, ${ }^{1}$ \\ Thomas Bochmann ${ }^{4}$
}

'Department of Child Health, University of Southampton and University Hospital Southampton NHS Foundation Trust, Southampton, UK ${ }^{2}$ Research Design Service, University of Southampton, Southampton, UK

${ }^{3}$ Trauma and Orthopaedics, University Hospital Southampton NHS Foundation Trust, Southampton, UK ${ }^{4}$ Department of Medicine, University of Southampton, Southampton, UK

\section{Correspondence to}

Nicholas M P Clarke Department of Child Health, Southampton General Hospital, Southampton S016 6YD, UK:

ortho@soton.ac.uk

Received 27 September 2011 Accepted 13 January 2012 Published Online First 12 March 2012

\section{ABSTRACT \\ Objectives The authors report the results of a selective ultrasound screening programme for congenital dislocation of the hip (CDH) over a period of 20 years, with the aim of defining the rate of screening, conservative treatment and late presentation requiring surgery. \\ Methods All neonates born from June 1988 to December 2008 (inclusive) were included in the prospective cohort, with a minimum follow-up of 12 months. All underwent an early clinical examination of the hips and those with clinical instability were referred for ultrasound at 2 weeks; those with risk factors were sonographically examined at 6 weeks. Risk factors were defined as breech presentation, family history or foot deformity.}

Results 107440 live births were clinically examined, 20344 (18.9\%) were referred for ultrasound assessment at either 2 weeks (due to clinical signs) or 6 weeks (due to risk factors). 774 (3.8\%) were diagnosed with dysplasia with a crude overall treatment rate of 7.2 per 1000 live births. 37 (0.34 per 1000) presented late, that is, after 12 weeks of age; none had detectable clinical signs or risk factors. There were no false negatives. Conclusion Elective screening for developmental dysplasia of the hip in association with one stop treatment and monitoring is an effective programme. The number of infants referred increased statistically significantly year on year over the study period and generated more activity. Pavlik harness treatment rates remained acceptable and steady over the period, despite the increase in referrals. The incidence of late presenting cases ranged from 0 to 4 per year, with no secular trend and there were no ultrasound false negatives.

\section{INTRODUCTION}

Congenital dislocation of the hip (CDH), or developmental dysplasia of the hip (DDH), encompasses a spectrum of hip pathology, including frank dislocation, subluxation and joint laxity. The term $\mathrm{DDH}$ takes into account the concept that abnormalities may develop with growth and altered development of the hip, arising primarily as a result of hip instability. ${ }^{1}$ This instability, if left untreated, may lead to a variable degree of hip dysplasia.

The incidence of neonatal hip instability is between 15 to 20 per 1000 live births, ${ }^{2}$ but the incidence of frank dislocation is $1-2$ per 1000 live births in an unscreened population, suggesting that in most cases spontaneous resolution occurs. $^{3}$

\section{What is already known on this topic}

Clinical screening for congenital dislocation of the hip (CDH) has proved unreliable. Comprehensive ultrasound screening is used in Europe successfully with significant economic consequences. Selective screening is used as a compromise.

\section{What this study adds}

This study confirms that selective ultrasound screening is successful in terms of surveillance with a very low late presentation rate and moderate economic implications.

The early diagnosis of $\mathrm{DDH}$ is vital as timely initiation of appropriate treatment minimises the associated risk of early osteoarthritis. ${ }^{4-7}$ Litigation concerning missed diagnosis of DDH is increasing. ${ }^{8}$ Atrey et al ${ }^{p}$ examined the National Health Service Litigation Authority over a 10-year period and found that DDH was the third most common cause in paediatric orthopaedics with an average cost of $£ 48534$.

The National Joint Registry of England and Wales suggests that the majority of hip replacements for DDH are performed below the age of 60 and this is reflected in other countries. ${ }^{10}$ This affects a working population with the increased likelihood of requiring multiple revision procedures and it represents a significant socioeconomic burden.

The reliability of clinical tests has remained elusive. However, since the introduction of ultrasound for diagnosis, there has been a fall in the surgical rates, ${ }^{11-15}$ leading to a reduction in costs of surgical treatment and our own preultrasound surgical rate of $1.28 / 1000^{3}$ reflects this. Ultrasound is more sensitive and specific than the clinical examnation tests of Ortolani ${ }^{16}{ }^{17}$ and Barlow, ${ }^{2}$ as these tests depend on the experience of the examiner ${ }^{18}$ and may be associated with higher false negatives. ${ }^{19}$

Screening programmes for DDH satisfy the prerequistes as described by Wilson and Jungner ${ }^{20}$ and meet the criteria of the UK National Screening Committee. ${ }^{21} \mathrm{DDH}$ is an important health problem and ultrasound is a reliable, non-invasive and 
low-risk test for diagnosis when performed at the appropriate time, in association with facilities for treatment.

There are several economic analyses of screening with ultrasound for DDH in the literature, ${ }^{12}{ }^{22-27}$ but the overall effectiveness of both comprehensive and selective screening programmes remains controversial.

We report our experience of using a programme of universal screening by clinical examination augumented by selective ultrasound, its effectiveness and its practice and pattern in one institutional practice over 20 years, supervised by the senior author.

\section{METHODS}

All infants born from June 1988 to December 2008 (inclusively) at the Princess Anne Maternity Hospital, Southampton, UK were included in the prospective cohort with a minimum follow-up of 12 months.

\section{Screening and one stop treatment}

Our protocol has been previously described ${ }^{3}$ and is illustrated in the algorithm shown in figure 1A. All neonates have a comprehensive postnatal examination by paediatric staff within $72 \mathrm{~h}$ of birth, including a full history to identify risk factors, using the Galeazzi, Ortolani and Barlow tests for hip instability or dislocation. All hip abnormalities are recorded, including instability, hip click or clunk and apparent limitation of abduction.

Babies with clinical instability are placed in double nappies and referred to the Hip Screening Clinic to attend at 10-16 days of age. Those with risk factors alone are seen at 6 weeks of age. Risk factors were defined as breech presentation, family history and foot deformity. Babies that are free of signs and risk factors will usually undergo the routine 6-week check up by their GP and health visitor. The clinical team includes two advanced nurse practitioners, who can be contacted at all times by local GPs, who have been continuously educated. Thus, if an infant is found to have missed risk factors or any hip abnormality they are referred to the hip screening clinic, which is open access, as a matter of urgency. (figure 1B)

The hip screening clinic occurs weekly and the Clarke et al ${ }^{28}$ sonographic method is used, which includes static and dynamic assessment with grading made in terms of location and stability.

Infants with clinically and sonographically stable hips are discharged from the clinic at 6 weeks old. Hips graded as 'concentrically reduced but subluxable', 'subluxed but reducible' or 'irreducibly dislocated' are placed directly into a Pavlik harness on a full-time basis. Treatment is then reviewed weekly until the hip reduces or until the treatment is deemed to be failing. If the Pavlik harness treatment is successful the infant is weaned after 6 weeks of splintage, over a further 6 weeks with weekly ultrasounds. Follow-up continues initially with ultrasound and subsequently radiographs after the ossific nucleus appears in the femoral head, up to the age 5 years.

In cases where the harness fails to reduce the hip after a maximum of 3 weeks, the child is entered into the surgical programme. Surgery is postponed until either an ossific nucleus is present in the femoral head or the infant is 13 months old, to reduce the risk of avascular necrosis. ${ }^{29}$

\section{Late presentations}

We define presentation as late when it occurs after 12 weeks of age. Pavlik harness treatment is increasingly unlikely to

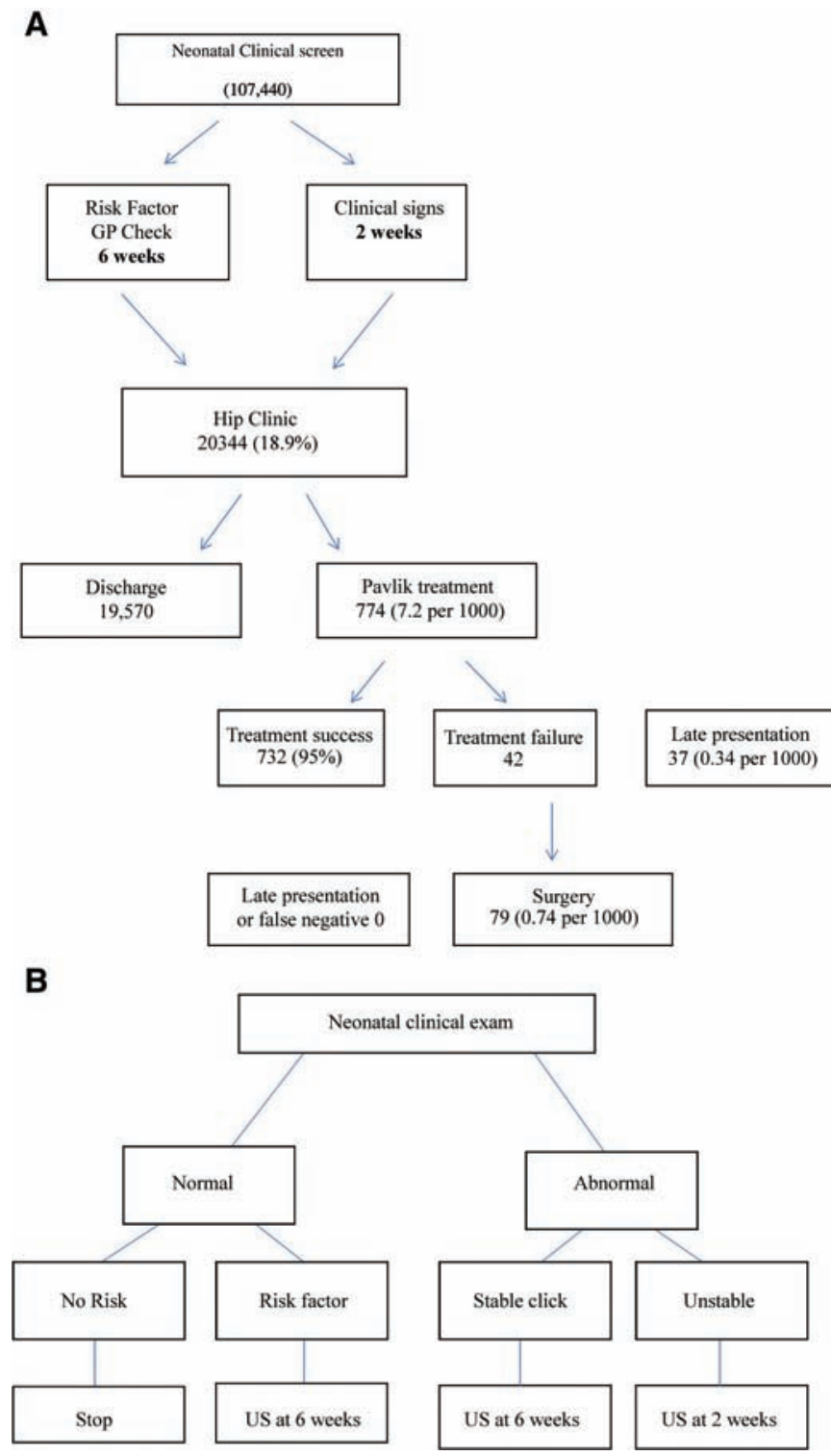

Figure 1 Screening and one stop treatment protocol.

concentrically reduce a displaced dysplastic hip and these infants are placed directly into the surgical programme. ${ }^{30}$

\section{Surgery}

Surgery was undertaken for failed Pavlik harness treatment and late presentation. Closed reduction was attempted after an adductor tenotomy, but if concentric reduction was not possible, open reduction was performed.

Closed reductions were placed in a spica cast for 6 weeks, followed by x-ray review and a further 6 weeks in a spica. Open reductions spent a single 6 -week period in a spica followed by conversion to abduction broomstick plaster.

Patients remain under clinical and radiological review until skeletal maturity.

\section{Costs}

Costs were calculated from Clegg et al $l^{12}$ and Atrey et al for screening, treatment and potential litigation. This framework was then applied to the results of the treatment protocol. As the costs from Clegg et al are from 1999 they should be used 
only as units for comparison, the '1999 index', as opposed to current economic valuations. To clarify this point all '1999 index' values are referred to as units.

Failure of conservative treatment is not usually the subject of legal action. Missed diagnosis or delayed referral to the hip screening clinic may be open to legal investigation and costs may be incurred.

Atrey's study ${ }^{31}$ published a rate of just 2.4 cases per year of litigation for DDH, however this is not consistent with the senior author's personal experience of exposure to 10 cases per year. Calculations are based on an estimate that 50 cases per year will progress to legal action in the UK, and therefore, an estimated $50 \%$ of late presentations will result in resolved legal action with the average cost of $£ 48534$ (2010 currency).

The cost framework is presented in table 1.

\section{Data collection}

Data for births from June 1988 (when the programme was initiated) to December 2008 were collected from the prospective records of the Princess Anne Maternity Hospital. Data for all infants referred to the hip screening clinic were prospectively recorded in a standard format in logbooks and the medical notes. Data for the surgical cases were also recorded prospectively in a standard format in the medical notes and these

\section{Table 1 Cost framework}

\begin{tabular}{lr}
\hline Aspect of protocol & Cost (f) \\
Ultrasound screening/clinic & 110 \\
Pavlik harness & 53 \\
Primary closed reduction (unilateral) & 2314 \\
Primary closed reduction (bilateral) & 2875 \\
Primary open reduction (unilateral) & 3286 \\
Primary bilateral (one open, one closed) & 3426 \\
Secondary open reduction (unilateral) & 1392 \\
Pelvic osteotomy (preceded by arthrogram) & 1853 \\
Litigation/case & 48534
\end{tabular}

Cost framework derived from Clegg et $\mathrm{a}^{12}$ and Atrey et al. ${ }^{31}$ All costs are inclusive of inpatient stay, theatre time, staff time, investigations and materials used. cases were identified from the theatre logbooks and admission records of the paediatric orthopaedic ward. The data were collated and stored in encrypted files on a password protected computer. Data collected included births per year, ultrasound screening numbers and findings, Pavlik harness treatments, late presentations and surgical rate.

\section{Statistical analysis}

The descriptive epidemiology of the Southampton Screening Protocol was obtained. Variations in rates of selective ultrasound screening (USS), diagnosis and treatment over the 20 -year period were explored using Poisson regression.

\section{Exclusions}

All infants born outside the Southampton district, home births, and all infants with neuromuscular or syndromic conditions, termed teratological dysplasia, were excluded.

\section{RESULTS}

A total of 107440 live births occurred at the Princess Anne Maternity Hospital during the 20 -year period of the study and 20344 (18.9\%) infants were referred for selective ultrasound assessment at either 2 weeks (due to clinical signs) or 6 weeks (due to risk factors alone or physical signs) (figure 1). Of these infants, 774 (3.8\%) were diagnosed with dyplasia and entered the treatment programme, with a crude overall treatment rate of $7.2 / 1000$ live births $(0.72 \%$ ) (figure 2 ). Pavlik harness treatment had an overall success rate of $94.6 \%$ with 732 infants responding to treatment; the remaining 42 infants were referred to the surgical programme following failed Pavlik treatment (figure 3). A further 37 (0.34 per 1000; $0.034 \%)$ infants presented late (ie, after 12 weeks of age) and were also referred to the surgical programme. None of these infants had clinical signs or risk factors at birth. The combined surgical rate arising from failed Pavlik treatment and late presentation was $0.74 / 1000(0.074 \%)$ live births $(\mathrm{N}=79)$ (figure 4$)$.

Of the 19570 infants discharged from the USS clinic with 'normal' hips, none presented to the service again, indicating a false-negative rate of DDH detection by USS of $0 \%$ and also

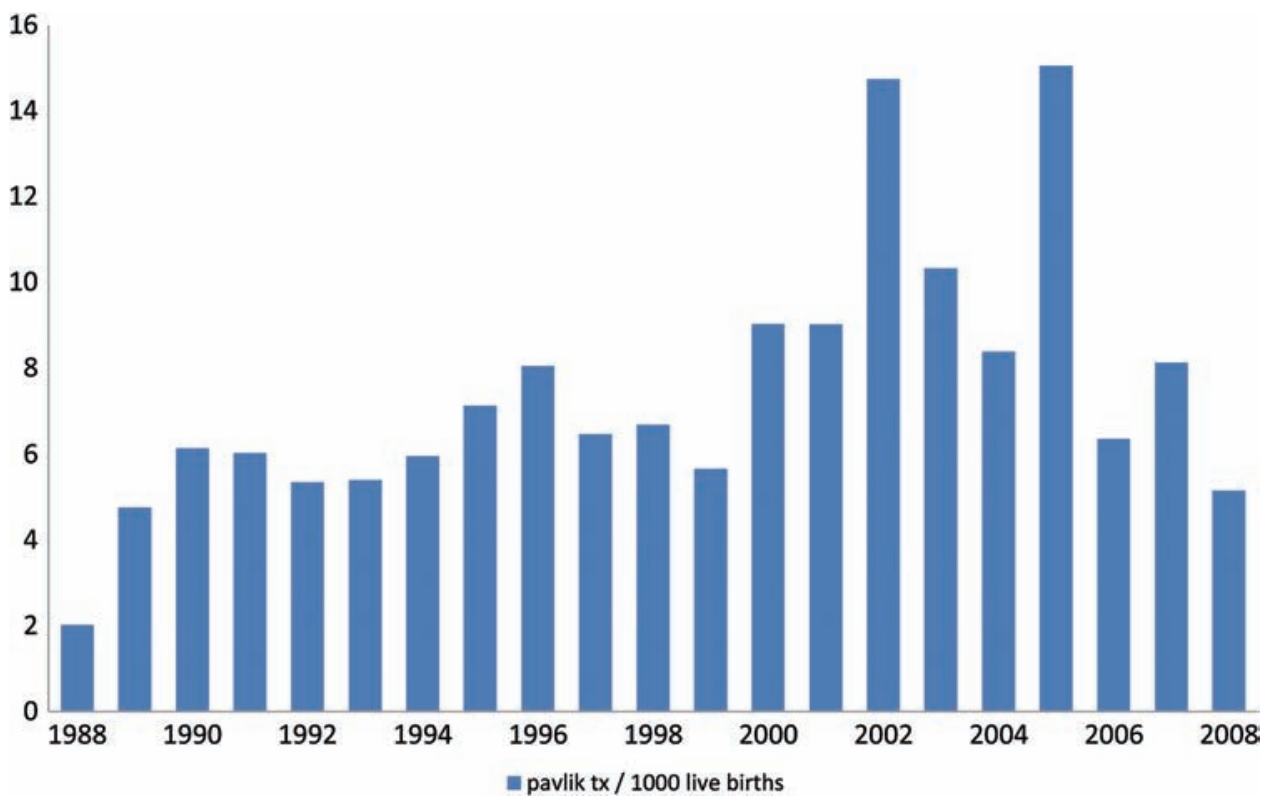

Figure 2 Pavlik harness treatment rate for the period June 1988-December 2008. 


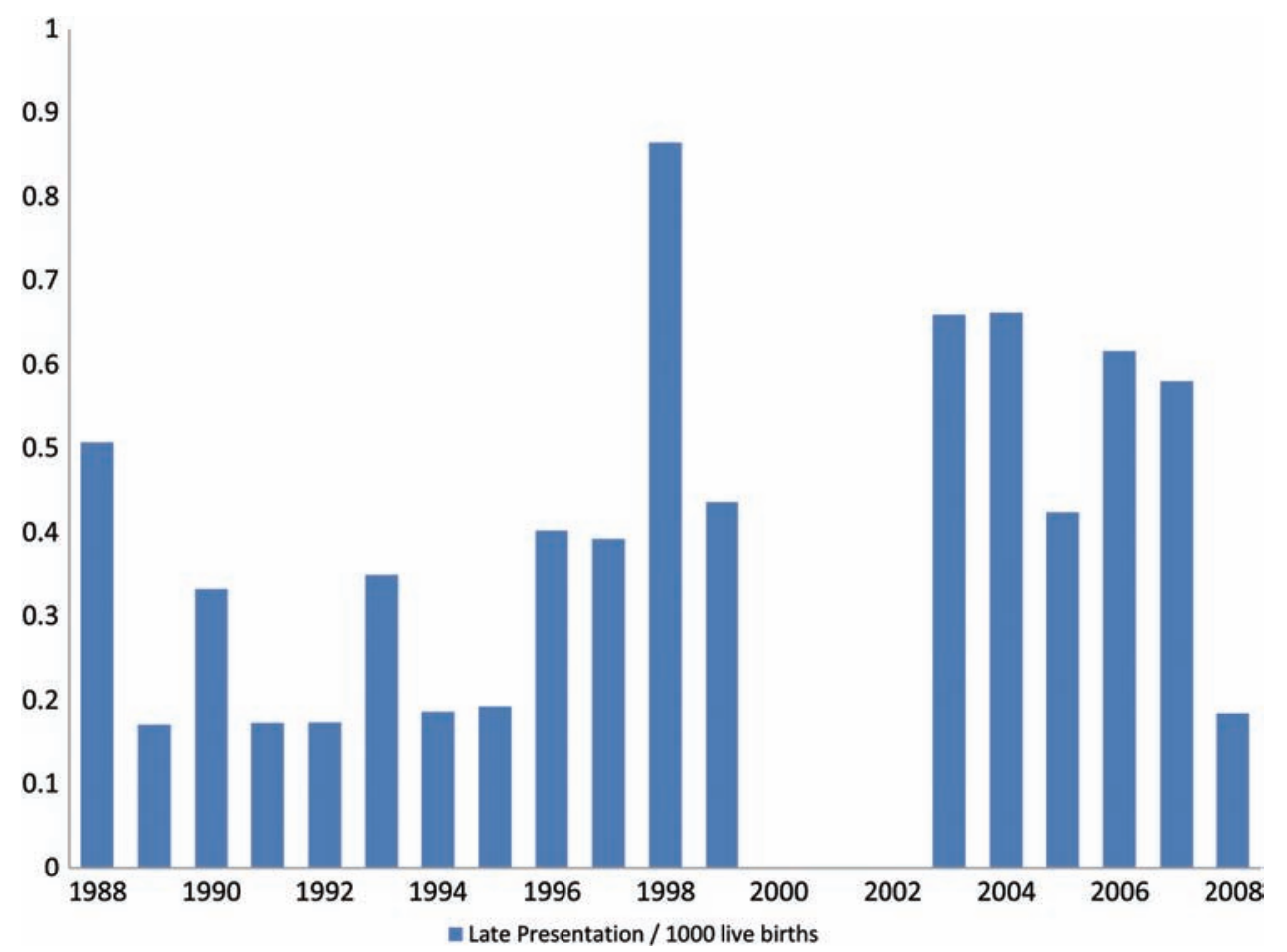

Figure 3 Late presentation rate for the period June 1988-December 2008.

a late presentation rate of $0 \%$ following USS at 6 weeks across the 20-year period.

\section{Secular trends over the $\mathbf{2 0}$-year study period}

The number of infants referred for selective ultrasound assessment increased statistically significantly over time such that, on average, $5 \%$ more patients were referred year on year (figure 5). This increase remained even after the birth rate was accounted for (incidence rate ratio per year 1.05, 95\% CI 1.05 to $1.06, \mathrm{p}<0.001$, Poisson regression model).

Treatment by Pavlik harness remained steady over the 20 -year period despite the increase in referral to selective USS (incidence rate ratio $1.00,95 \%$ CI 0.9994 to $1.0003, p=0.58$ ) after birth rate and year were accounted for.

Non-response to Pavlik treatment ranged from 0 to 6 cases per year, but no secular trend was seen (incidence rate ratio $1.04,95 \%$ CI 0.99 to $1.09, \mathrm{p}=0.13$ ). Similarly, late presentation of $\mathrm{DDH}$ ranged from 0 to 4 cases per year, with no secular trend observed (incidence rate ratio $1.01,95 \%$ CI 0.96 to 1.07 , $\mathrm{p}=0.61$ ). Surgical treatment varied from 0 to 9 cases per year, but again no secular trend was seen (incidence rate ratio 1.03, $95 \%$ CI 0.99 to $1.07, \mathrm{p}=0.14$ ).

\section{DISCUSSION}

There is considerable debate in the literature as to whether screening for DDH should be practiced. In their review for the US Preventative Services Task Force in 2005, Shipman et a ${ }^{\beta 5}$ could not recommend that screening be adopted, as there is a lack of evidence of the effectiveness of intervention on functional outcomes. In their analysis of the literature, they explored several key questions that essentially summarise the controversies surrounding screening for DDH.

Screening is thought to lead to improved outcome on the premise of reducing the likelihood of surgery. The decline in surgical rates seen in our series compared with the prescreening era reflects this (0.74 vs 1.28 per 1000), and as our outcome measure for surgery is a lack of a concentric reduction at 12 weeks of age, this reduction in surgical rates is not due to an increased period of observation.

There are concerns that screening increases the false-positive rate, as the earlier the screening is done the more likely cases of instability will be detected. Therefore there will be a high rate of spontaneous resolution of instability and dysplasia, which will artificially increase the splintage rate. Our protocol therefore uses delayed selective ultrasound (at 6 weeks) for risk factors, so that these false positives are theoretically reduced.

Emphasis is placed on positive family history and breech presentation at delivery as pertinent risk factors in our protocol. The presence of these may be associated with more thorough examinations and more aggressive follow-up, which may again increase the numbers of false positives. Fear of litigation may be a contribution to our findings of the increasing number of infants referred for ultrasound assessment, and the ease of rapid access to the clinic. There is also reduced experience in nurse practitioner and in junior doctor grades because of modern working practices. The default position for the practitioner is referral for ultrasound assessment leading to the increasing numbers referred.

Advocates of screening suggest that early identification allows early treatment and intervention is less likely to be invasive and more likely to lead to a better outcome. There are several programmes of screening in the literature and these essentially fall under two basic categories: universal screening programmes with clinical examination and ultrasound, and selective ultrasound programmes which incorporate universal clinical examinations, with referral of infants with positive findings and infants at risk. ${ }^{32} 33$

Critics of screening ${ }^{35}$ suggest that ultrasound screening has poor interobserver and moderate intraobserver reliability and there is a considerable false-positive rate with overtreatment. This exposes these otherwise disease-free infants 


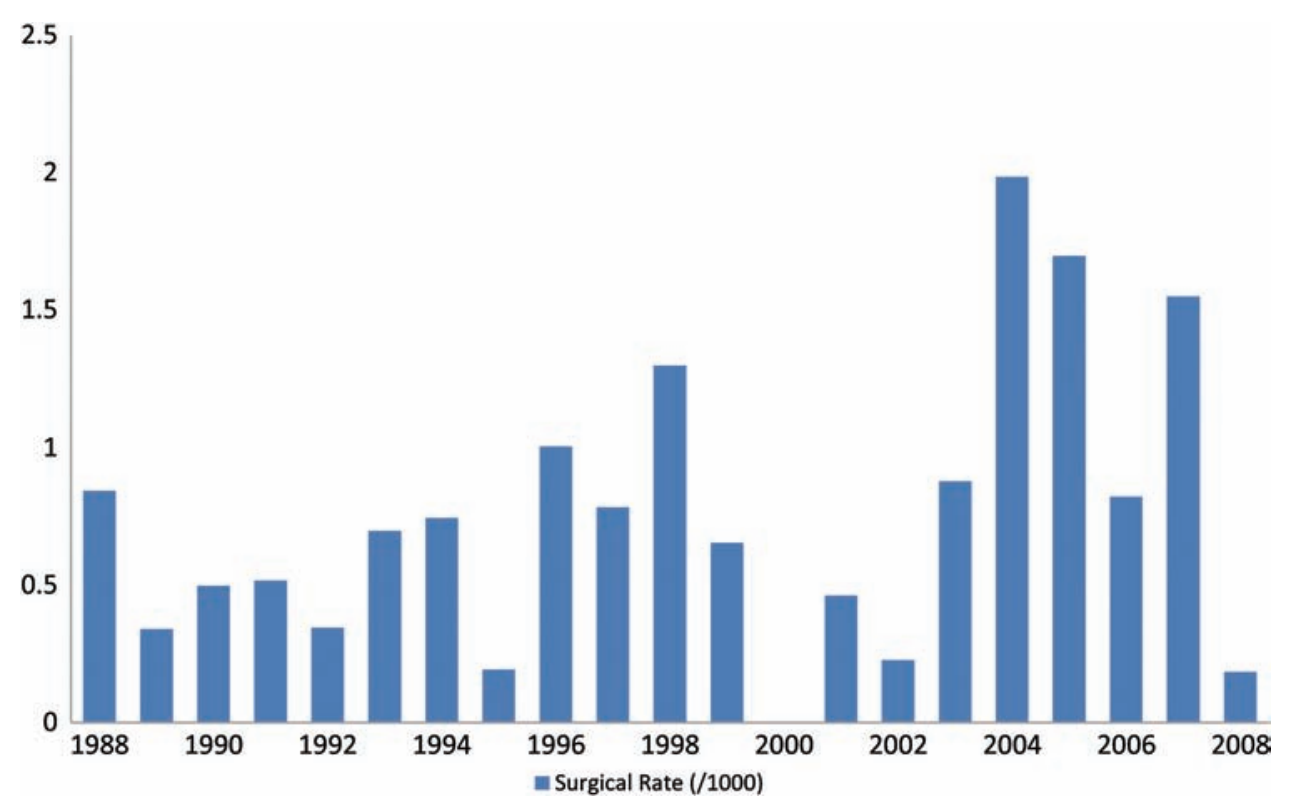

Figure 4 Surgical treatment rate for the period June 1988-December 2008.

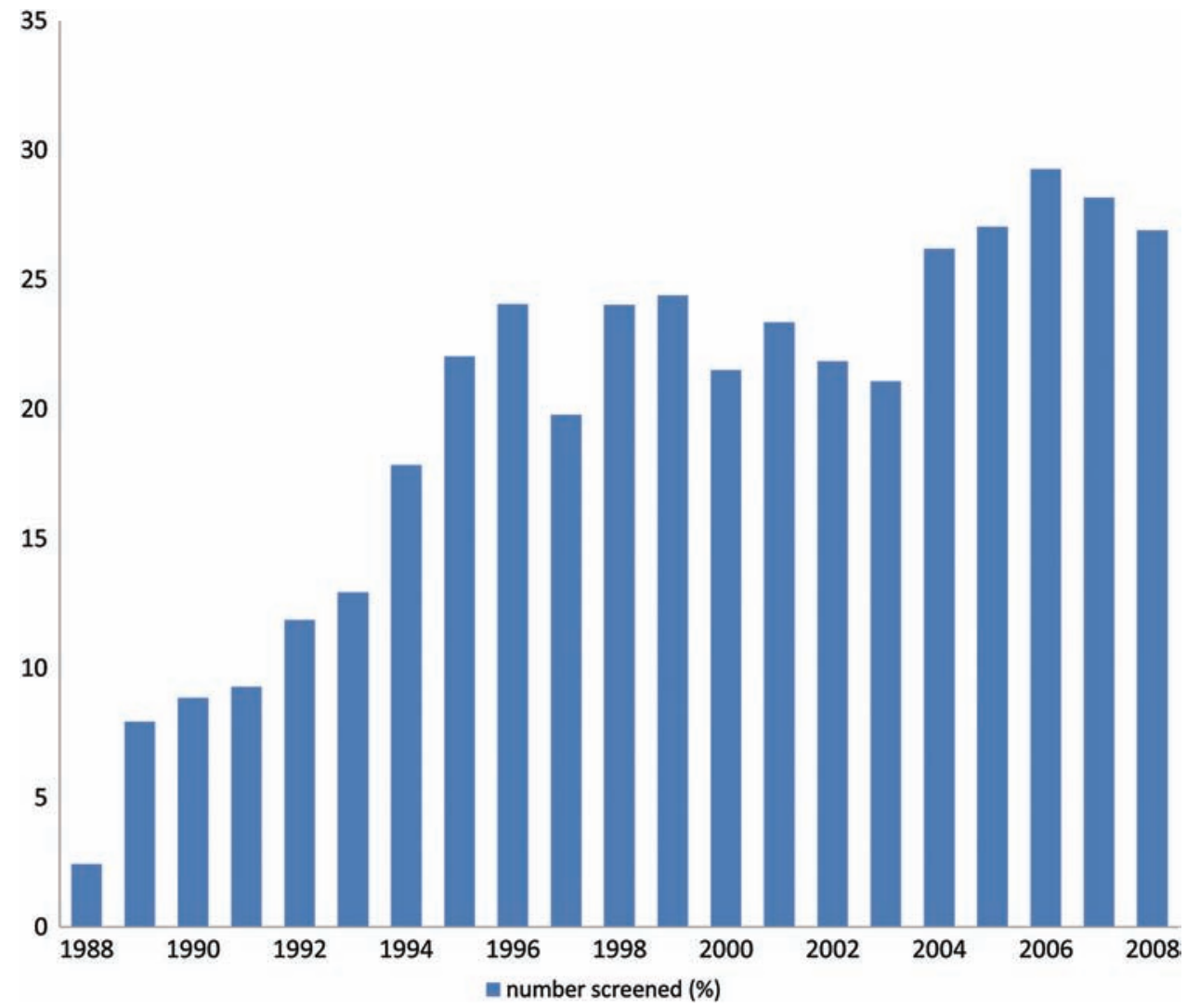

Figure 5 Percentage of infants undergoing ultrasound screening for the period June 1988-December 2008.

to the iatrogenic harms of avascular necrosis, femoral nerve palsies, undue parental anxiety, increased costs resulting from additional clinic visits and imaging, lost parental revenue from missed work and inefficient use of the clinician's time.

However, Mahan et a ${ }^{\beta 6}$ using decision analysis to evaluate the influences of screening on the probability of not developing an arthritic hip as a result of DDH, concluded that the optimum strategy was selective screening, when no screening, universal screening and selective screening programmes were compared. A recent meta-analysis concludes that there is insufficient evidence to recommend a method of screening. ${ }^{37}$

We use the Clarke et al ${ }^{28}$ method of ultrasound assessment which is less complicated than the Graf method. ${ }^{38}$ It has static and dynamic components and therefore is less vulnerable to intraobserver and interobserver variability. Our use of selective delayed ultrasound screening decreases the false-positive 
rate and the cost incurred with these over-diagnoses, as well as the false negatives that an examination-alone programme would incur. Our results demonstrate that despite the higher number of patients referred, late presentations did not increase until 2003. This increase may be due to increased numbers of births from mothers that immigrated from higher DDH endemic regions in Europe, although we lack complete data on the mothers' origins. This phenomenon has also been reported in Austria (R Graf, personal communication). Any screening programme should follow the laws of screening as described by Wilson and Jungner ${ }^{20}$ and our selective screening programme does this, as well as provide a one stop clinic where treatment is provided immediately on diagnosis.

A recent study from Austria compared the cost effectiveness of universal ultrasound screening compared with clinical examination alone.. ${ }^{39}$ A $75.9 \%$ decrease in the total number of surgical interventions was noted after the introduction of ultrasound. However, the overall cost of screening and treatment of DDH increased mainly due to the ultrasound programme itself. Our programme therefore is effectively a compromise in terms of cost effectiveness and resource availability, with a reduction in late presenting cases in the context of a low primary treatment rate $(7.2$ per 1000) compared with universal screening generating a treatment rate of up to 60 per 1000 .

For this reason our programme should perhaps be deemed 'surveillance'. There is a consensus, however, that the condition is important and that treatment and diagnostic facilities should be available and treatment is acceptable to the patient.

There is no generally accepted opinion on what hip abnormality (clinical or sonographic) should be treated and for what length of time.

Our programme showed a higher late presentation rate than the universal screening programmes described in the literature $^{40}(0.34$ vs 0 per 1000).

Our cost framework has shown that the ultrasound clinic has less cost, as previously reported, ${ }^{41}$ than the necessary surgical interventions, which have been reduced in number over the study period.

We conclude that:

- Selective screening for DDH in association with one stop treatment and monitoring is an effective programme but a compromise.

- 'Surveillance' is probably a better term than screening.

- The number of infants referred increased statistically significantly year on year over the study period and generated more activity.

- Pavlik harness treatment rates remained acceptable and steady over the period despite the increase in referrals.

- Late presenting cases ranged from 0 to 4 per year $(0.34$ per 1000) with no secular trend.

- There were no ultrasound false negatives.

Contributors All authors have read and approved the manuscript that they have contributed to and believe it to be honest work and are able to verify the validity of the results reported.

Competing interests None.

Provenance and peer review Not commissioned; externally peer reviewed.

\section{REFERENCES}

1. Klisic PJ. Congenital dislocation of the hip —a misleading term: brief report. J Bone Joint Surg Br 1989;71:136.

2. Barlow TG. Congenital dislocation of the hip: early diagnosis and treatment. Lond Clin Med J 1964;13:47-58.

3. Boeree NR, Clarke NM. Ultrasound imaging and secondary screening for congenital dislocation of the hip. J Bone Joint Surg Br 1994:76:525-33.
4. Jacobsen S, Sonne-Holm S, Søballe K, et al. Hip dysplasia and osteoarthrosis: a survey of 4151 subjects from the Osteoarthrosis Substudy of the Copenhagen City Heart Study. Acta Orthop 2005;76:149-58.

5. Fujii M, Nakashima Y, Jingushi S, et al. Intraarticular findings in symptomatic developmental dysplasia of the hip. J Pediatr Orthop 2009;29:9-13.

6. Engesæter IØ, Lehmann T, Laborie LB, et al. Total hip replacement in young adults with hip dysplasia: age at diagnosis, previous treatment, quality of life, and validation of diagnoses reported to the Norwegian Arthroplasty Register between 1987 and 2007. Acta Orthop 2011;82:149-54.

7. Jacobsen S, Sonne-Holm S. Hip dysplasia: a significant risk factor for the development of hip osteoarthritis. A cross-sectional survey. Rheumatology (Oxford) 2005:44:211-18

8. McAbee GN, Donn SM, Mendelson RA, et al. Medical diagnoses commonly associated with pediatric malpractice lawsuits in the United States. Pediatrics 2008;122:e1282-6.

9. Atrey A, Gupte CM, Corbett SA. Review of successful litigation against English health trusts in the treatment of adults with orthopaedic pathology: clinical governance lessons learned. J Bone Joint Surg Am 2010;92:e36.

10. Furnes 0, Lie SA, Espehaug B, et al. Hip disease and the prognosis of total hip replacements. A review of 53,698 primary total hip replacements reported to the Norwegian Arthroplasty Register 1987-99. J Bone Joint Surg Br 2001;83:579-86.

11. Graf R. The use of ultrasonography in developmental dysplasia of the hip. Acta Orthop Traumatol Turc 2007;41 Suppl 1:6-13.

12. Clegg J, Bache CE, Raut VV. Financial justification for routine ultrasound screening of the neonatal hip. J Bone Joint Surg Br 1999;81:852-7.

13. Wirth T, Stratmann L, Hinrichs F. Evolution of late presenting developmental dysplasia of the hip and associated surgical procedures after 14 years of neonatal ultrasound screening. J Bone Joint Surg Br 2004;86:585-9.

14. Woolacott NF, Puhan MA, Steurer J, et al. Ultrasonography in screening for developmental dysplasia of the hip in newborns: systematic review. BMJ 2005; 330:1413.

15. von Kries R, Ihme N, Oberle D, et al. Effect of ultrasound screening on the rate of first operative procedures for developmental hip dysplasia in Germany. Lancet 2003;362:1883-7.

16. Dickson JW. Pierre Le Damany on congenital dysplasia of the hip. Proc R Soc Med 1969:62:575-7.

17. Bennet GC. Screening for congenital dislocation of the hip. J Bone Joint Surg Br 1992:74:643-4.

18. Macnicol MF. Results of a 25-year screening programme for neonatal hip instability. J Bone Joint Surg Br 1990;72:1057-60.

19. Lipton GE, Guille JT, Altiok H, et al. A reappraisal of the Ortolani examination in children with developmental dysplasia of the hip. J Pediatr Orthop 2007;27:27-31.

20. Wilson JM, Jungner YG. Principles and practice of mass screening for disease Bol Oficina Sanit Panam 1968:65:281-393.

21. Committee UNS, 2011. http://www.screening.nhs.uk/criteria (accessed 12 Feb 2012)

22. Rosendahl K, Markestad T, Lie RT, et al. Cost-effectiveness of alternative screening strategies for developmental dysplasia of the hip. Arch Pediatr Adolesc Med 1995;149:643-8.

23. Geitung JT, Rosendahl K, Sudmann E. Cost-effectiveness of ultrasonographic screening for congenital hip dysplasia in new-borns. Skeletal Radiol 1996;25:251-4.

24. Bralić I, Vrdoljak J, Kovacić L. Ultrasound screening of the neonatal hip: costbenefit analysis. Croat Med J 2001;42:171-4.

25. Brown J, Dezateux C, Karnon J, et al. Efficiency of alternative policy options for screening for developmental dysplasia of the hip in the United Kingdom. Arch Dis Child 2003;88:760-6

26. Dezateux C, Brown J, Arthur R, et al. Performance, treatment pathways, and effects of alternative policy options for screening for developmental dysplasia of the hip in the United Kingdom. Arch Dis Child 2003;88:753-9.

27. Elbourne D, Dezateux C, Arthur R, et al. Ultrasonography in the diagnosis and management of developmental hip dysplasia (UK Hip Trial): clinical and economic results of a multicentre randomised controlled trial. Lancet 2002;360:2009-17.

28. Clarke NM, Harcke HT, McHugh P, et al. Real-time ultrasound in the diagnosis of congenital dislocation and dysplasia of the hip. J Bone Joint Surg Br 1985;67:406-12

29. Clarke NM, Jowett AJ, Parker L. The surgical treatment of established congenital dislocation of the hip: results of surgery after planned delayed intervention following the appearance of the capital femoral ossific nucleus. J Pediatr Orthop 2005;25:434-9

30. Harding MG, Harcke HT, Bowen JR, et al. Management of dislocated hips with Pavlik harness treatment and ultrasound monitoring. J Pediatr Orthop 1997; 17:189-98

31. Atrey A, Nicolaou N, Katchburian M, et al. A review of reported litigation against English health trusts for the treatment of children in orthopaedics: present trends and suggestions to reduce mistakes. J Child Orthop 2010;4:471-6.

32. Graf R. New possibilities for the diagnosis of congenital hip joint dislocation by ultrasonography. J Pediatr Orthop 1983;3:354-9. 
33. Graf R. Hip sonography: 20 years experience and results. Hip Int 2007:17 Suppl 5:S8-14.

34. Holen KJ, Tegnander A, Bredland T, et al. Universal or selective screening of the neonatal hip using ultrasound? A prospective, randomised trial of 15,529 newborn infants. J Bone Joint Surg Br 2002;84:886-90.

35. Shipman SA. Risk management and developmental dysplasia of the hip: primum non nocere. Pediatrics 2009;123:e546.

36. Mahan ST, Katz JN, Kim YJ. To screen or not to screen? A decision analysis of the utility of screening for developmental dysplasia of the hip. J Bone Joint Surg Am 2009:91:1705-19.

37. Shorter D, Hong T, Osborn DA. Screening programmes for developmental dysplasia of the hip in newborn infants. Cochrane Database Syst Rev 2011;9:CD004595.
38. Graf R. The diagnosis of congenital hip-joint dislocation by the ultrasonic Combound treatment. Arch Orthop Trauma Surg 1980;97:117-33.

39. Marks DS, Clegg J, al-Chalabi AN. Routine ultrasound screening for neonatal hip instability. Can it abolish late-presenting congenital dislocation of the hip? J Bone Joint Surg Br 1994;76:534-8.

40. Thaler M, Biedermann R, Lair J, et al. Cost-effectiveness of universal ultrasound screening compared with clinical examination alone in the diagnosis and treatment of neonatal hip dysplasia in Austria. J Bone Joint Surg Br 2011; 93:1126-30.

41. Gray A, Elbourne D, Dezateux C, et al. Economic evaluation of ultrasonography in the diagnosis and management of developmental hip dysplasia in the United Kingdom and Ireland. J Bone Joint Surg Am 2005;87:2472-9. 